\title{
Artificial Intelligence-Based Business Communication: Application for Recruitment and Selection
}

\author{
Mina Son ${ }^{1}$, Hyeonju Lee ${ }^{1}$, Hyejung Chang ${ }^{2}$ \\ ${ }^{1}$ MIDAS Information Technology Co., Ltd., Seongnam, Korea \\ ${ }^{2}$ Kyung Hee University, Seoul, Korea
}

Objectives: Artificial intelligence (AI) has become an increasingly important issue with the rise of the Fourth Industrial Revolution. In human resource (HR) management, one of the most important tasks is the recruitment of qualified employees. This paper examines inAIR (AI for Recruiting), a recent recruitment integration solution, to explore how AI technology enables new communication channels in the recruitment process.

Methods: The inAIR is an online system that manages the recruitment process, which includes but is not limited to document reviews, personality tests, interview screenings, and judgement suggestions. The AI-based interview step demonstrates how inAIR uses $\mathrm{AI}$ and machine learning.

Results: The value of the AI interview is that it maximizes the communication channels between the applicants and the HR manager. While the HR team can now receive immediate results equivalent to those usually reached by interviewing thousands of applicants, applicants can still convey their passion, experience, and abilities beyond what has been expressed in their documents. The inAIR is an efficient tool in terms of the time required: An applicant takes about an hour to complete an AI interview, and the system takes no more than an hour to analyze all the data from thousands of applicants.

Conclusions: AI has been adopted in the recruitment process as well as processes that evaluate employee experience, train employees in various job skills, and make decisions necessary for business management. With the development of the Internet and AI technology, the communication channels between applicants and HR managers have been expanded, allowing a variety of processes to run much more efficiently.

Key Words: Human Resource Management, Recruitment, Artificial Intelligence, Interview

Received: Feb 3, 2019 Revised: Mar 4, 2019 Accepted: Mar 7, 2019

Corresponding author: Hyejung Chang

School of Management, Kyung Hee University, 26 Kyungheedae-ro, Dongdaemun-gu, Seoul 02447, Korea

Tel: +82-2-961-9432, E-mail: hjchang@khu.ac.kr

This is an Open Access article distributed under the terms of the Creative Commons Attribution Non-Commercial License (http://creativecommons.org/licenses/ by-nc/4.0/) which permits unrestricted non-commercial use, distribution, and reproduction in any medium, provided the original work is properly cited.

Copyright $(9) 2019$ Korean Association for Business Communication.

\section{Introduction}

Artificial intelligence (AI), a foundational element of the Fourth Industrial Revolution, has emerged as an important issue across all industries, and business management is no exception. AI and machine learning are now applied in areas of business such as marketing, human resource management (HRM), and manufacturing. In HRM, the technologies have been used to enhance employee experience, provide personalized vocational training, 
and analyze the various HR data to make decisions necessary for business management (Dorel \& Aleksandra, 2011). The application of these technologies to the HRM sector represents a unique case as the sector has been regarded as a human domain that explicitly deals with people (Lengnick-Hall, Lengnick-Hall, Andrade, \& Drake, 2009).

The use of AI technology in HRM has seen significant growth in multiple well-known settings. For example, IBM has reduced costs and improved operations in HRM system using Blue Matching, CogniPay, and Watson Career Coach (Lewis, 2019; Sheopuri \& Stachura, 2018). Blue Matching predicts mobile tasks based on the current workplace, salary level, role, and career level of its members. CogniPay supports managers to make better decisions by assessing employee performance and providing information such as compensation levels and market demand. Watson Career Coach is a career coaching system that allows employees to identify what is lacking in terms of their capacity. Amazon AI Recruitment Program, which recently received bad publicity for its negative bias against women, evaluates applicants' resumes to find applicants with a history similar to those of high performers, filtering out undesirable applicants (Gershgorn, 2018).

One of the most important tasks of an HRM department is the recruitment of qualified employees for the company. As very few applicants among those who submitted resumes are reviewed due to limitations of interviewer resources, applications and resumes are thus filtered based on the language proficiency, academic achievements, and test scores, and other data found in the submitted documents. Time and cost factors also eliminate many applicants before the actual interview stage. This phenomenon is particularly visible in open recruitment. If companies were able to thoroughly review documents and conduct in-depth interviews for each applicant, they would gain more information about the potential competencies of applicants and eliminate any chance of overlooking potentially valuable recruits.

Although the interview process has potential benefit immensely from the adoption of $\mathrm{AI}$ and machine learning, the use of these technologies has not yet been made popular. Interviewing is a process in which trained interviewers meet job seekers and evaluate how competent and enthusiastic applicants are based on communication that takes place over several minutes. Interviewing is thus considered a high-cost recruitment tool. If AI can help in this complex process, it will benefit both companies that make significant investments in their interview process and applicants who are not usually given opportunities due to the limitations of the interview process. Several companies have been trying to utilize AI in their interview process (Feloni, 2017), and this paper examines one such AI interview program developed by MIDAS IT (MIDAS Information Technology Co., Ltd.).

MIDAS IT is a South Korean company that develops simulation softwares for safety and economic analysis in the engineering field and holds a high global market share in the construction sector (https://www.midasoft.com/aboutus). MIDAS IT has recently introduced their AI technology to provide online services in various fields such as dementia diagnosis and management web solutions. In 2015, the company launched an online solution that makes possible the integrated management of the entire process of recruitment to the final selection of successful candidates (Cho, 2018). Their recruitment integration solution, called inAIR (AI for Recruiting), is a tool that enables the HR manager to communicate online with applicants and evaluate them through special programs developed by AI and machine learning (https:// www.midashri.com/intro/ai). In this paper, we examine inAIR to find how information technology (IT) enables new communication pathways in the employee recruitment process.

\section{Description of Recruitment System using Al}

\section{Communication Process between Applicant and HR Manager} The inAIR is a total recruitment system developed by MIDAS IT and has been adopted by various companies. It is an online system that comprehensively manages the recruitment process, including document reviews, personality tests, interview screenings, pass-failure judgements, and applicant guidance (Figure 1).

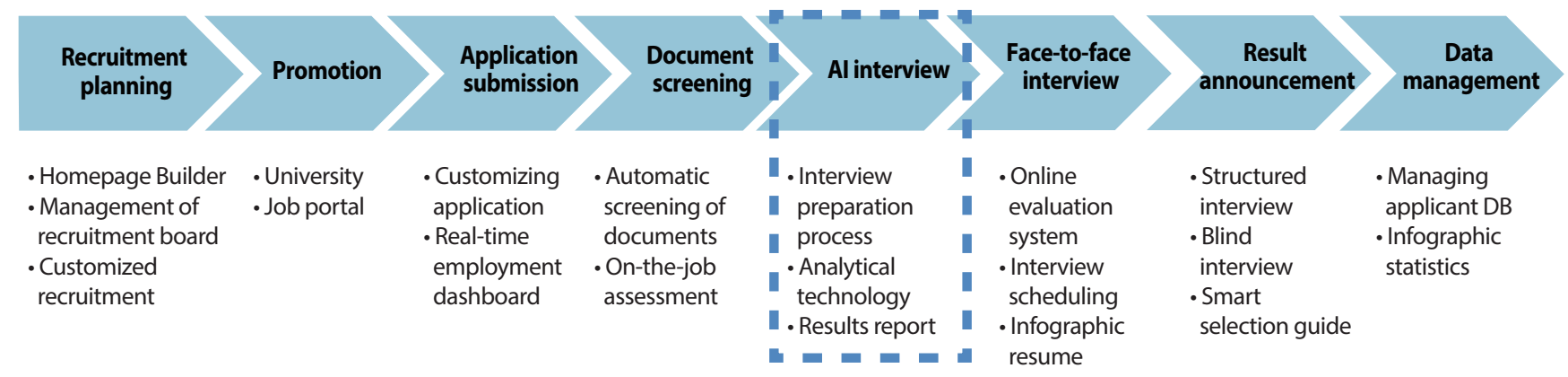

Figure 1. HR process using the inAIR system. Al, artificial intelligence; HR, human resource; $D B$, database. 
The AI Interview step is a unique feature demonstrating the use of AI technology. The AI interview process was developed to help HR departments recruit the best talent, as it reviews applicant competence more efficiently and on a wider scale, providing opportunities for applicants who could not otherwise reveal their strengths in their submitted documents.

The AI interview has applicants access a company's website, take automated interviews, and participate in games and surveys that assess their competency. This step is recommended immediately after the document screening stage as it helps the HR department review more applicants in a detailed fashion before the face-to-face interview. AI interview is thus a communication tool that connects the applicant and the recruiter as it is a tool for both interviews and evaluations.

The applicant can access the AI interview system and be interviewed at any place and any time during the period set by the HR manager. The system collects images from the applicant's responses to the questions and analyzes changes in facial expression, movement, voice, expression, and physiology. It also evaluates the interviewee's potential competency by analyzing the individual characteristics of the applicant when they respond to the game. After the applicant completes the AI interview, the HR manager can access a report summarizing the applicant's results.

\section{Al Interview Process Experienced by Applicants}

Applicants receive a link to access the $\mathrm{AI}$ interview website with a unique ID and entry code given to them in an e-mail. They can then access the website for an interview at a time of their choosing. When an applicant logs into the online interview site, the AI interview system guides the applicant through a pre-determined interview process (Figure 2).

As applicants access the interview using different personal computer systems, the website checks the applicant's system and verifies that the webcam and voice recognition work properly before the beginning of the interview. If the system meets all the requirements, the interview process begins by registering the applicant's face in order to prevent cases of someone else doing the interview for the candidate.

Interview questions are presented on the screen and spoken aloud by the program. After reading and listening to the question, the applicant is given a minute to prepare an answer and an explanation for that answer. Applicant are given one to two minutes to respond.

Interview questions are of different types. The beginning part of the AI interview consists of basic questions such as those in which applicants introduce themselves and state their motivation to apply. Surveys are then conducted to assess the applicant's opinions, and several games are played to evaluate competency based on neuroscience-based algorithms. In the second half of the interview process, individually-customized questions are asked based on the results of the first half of the AI interview. The applicant is then asked in-depth structured questions concerning the applicant's thoughts and experiences in particular situations. The company can also decide to add questions of their own, such as questions regarding job-specific skills or knowledge regarding the position. Screen shots of a typical interview process are shown in Figure 3.

\section{Al Interview Process Experienced by HR Managers}

HR managers receive the results of the applicants' AI interviews in three parts. The first is a video of the actual AI interview, which can be confirmed directly by both the HR manager and interviewers. Since it is difficult to know what kind of person an applicant is until the face-to-face interview, the HR manager can see through $\mathrm{AI}$ interview videos how passionate the applicant is for the job and how much related experience he or she has had. The manager can also check how the applicant structures his or her statements during the conversations. This kind of information is difficult to derive through documents. Pre-checking the AI interview videos reduces the time and effort that HR managers and interviewers would have had to spend choosing applicants from face-to-face interviews, increasing the efficiency of the interview process.

The remaining two parts are reports. One is a report that summarizes the interview results by analyzing and quantifying the video data collected during the interview, and the other is a detailed report that evaluates the applicant's abilities and predicts the performance and potential they showed through the games and surveys.

\section{Al Technology Used in Recruitment System}

\section{Data Collection and Analysis during the Al Interview Process}

During the AI interviews, the program analyzes changes in facial expression, voice, expression, and physiology by extracting various characteristic data from the image of the applicant answering the questions. These data are raw data of size and direction of facial muscle movements that replace human visual, auditory, and verbal functions.

The extracted raw data is analyzed and interpreted with various emotional expressions (Table 1). An additional function of the $\mathrm{AI}$ interview process is to provide an evaluation of the applicants by learning their behaviors. Based on the model derived from the evaluations given by the interviewers, the system provides the probabilities of the applicants' affinity, expressiveness, 


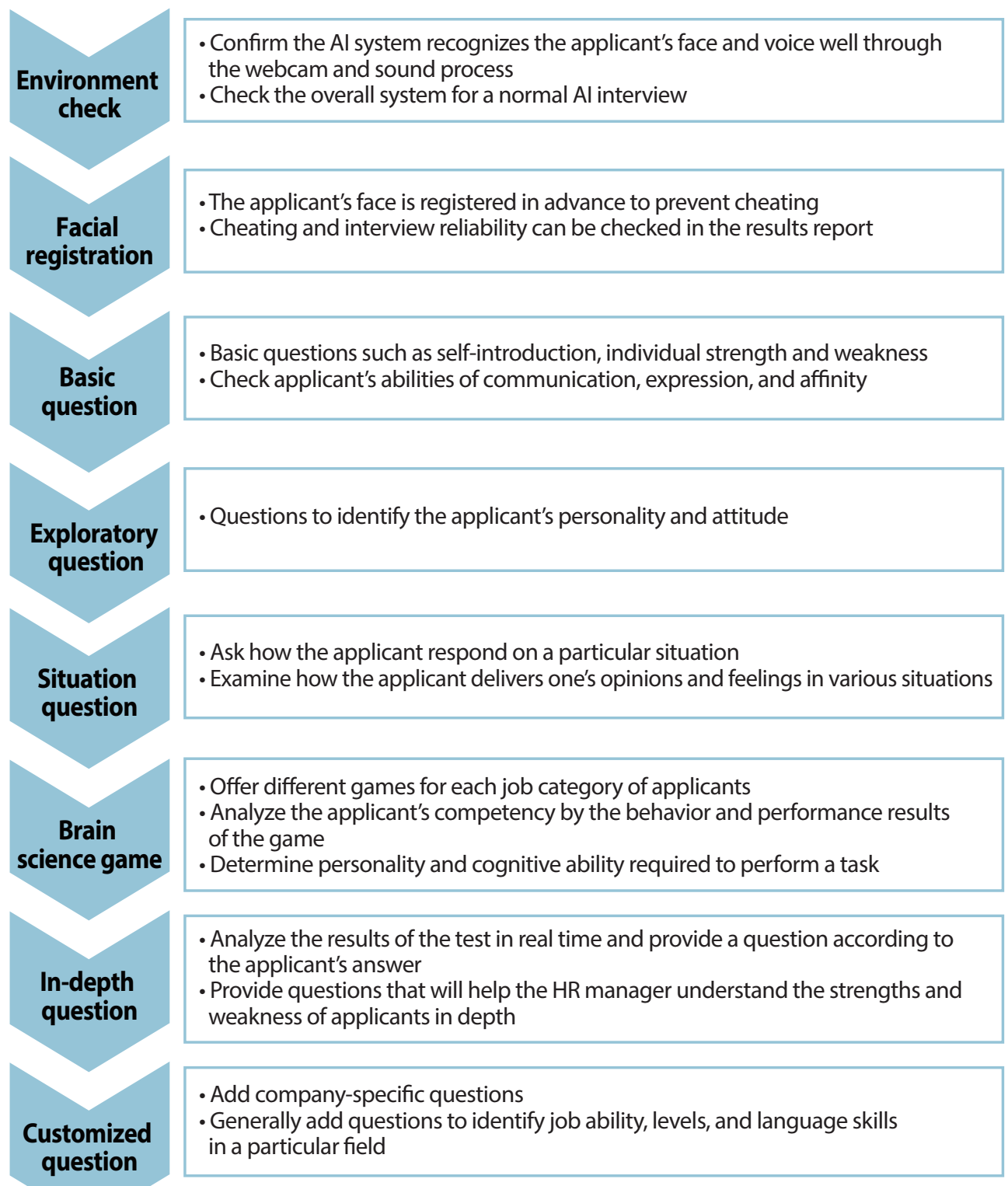

Figure 2. Al interview process experienced by applicants. Al, artificial intelligence.

and emotional delivery abilities, consistent with the judgments of the interviewer.

\section{Gamification for Evaluating Competency}

The AI interview includes game-based exploration to determine whether an applicant likely to produce high outcomes has characteristics similar to those of high-grade performers. The game is a new approach in recruitment and selection and is completely different from traditional tools or aptitude tests in terms of background theories, concepts, and measurement.

The competency evaluation game of the $\mathrm{AI}$ interview process is based on neuroscience theory. The prefrontal cortex area is the most developed area of the brain in humans and is largely utilized in social interactions involving internal motivation (Murayama et al., 2013) and higher cognitive function (Denny, Kober, Wager, \& Ochsner, 2012). Cognitive ability, in particular, which includes executive control, is known for its function in the dorsolateral prefrontal cortex predicts job performance (Higgins, Peterson, Pihl, \& Lee, 2007). This ability is therefore more valuable than general mental ability examinations in the selection scene as there are fewer adverse effects (Bosco, Allen, \& Singh, 2015).

MIDAS IT has developed computer game-based tasks used in the kind of neuroscience and cognitive psychology research 


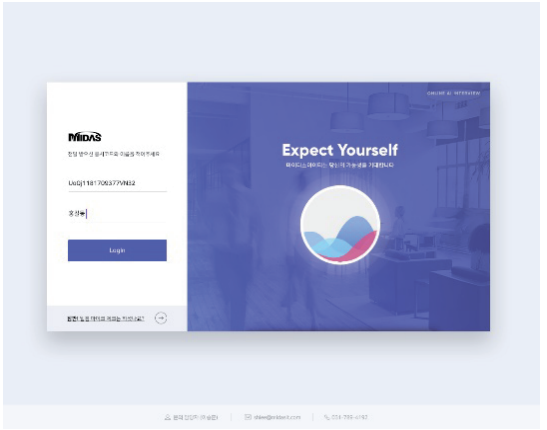

Log in

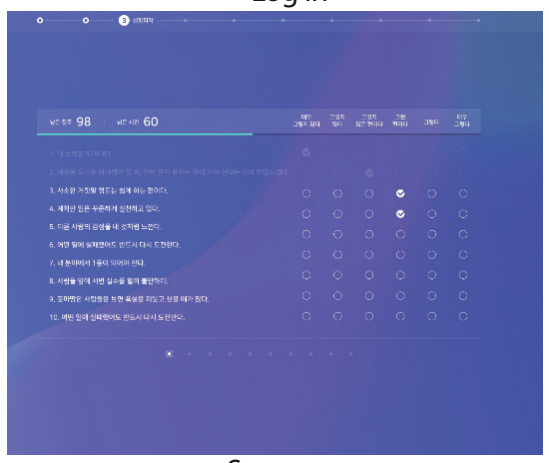

Survey

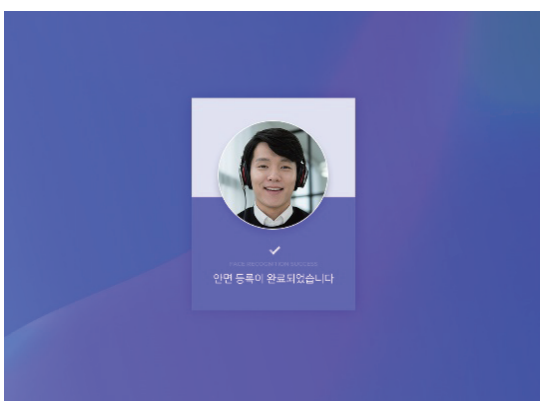

Facial registration

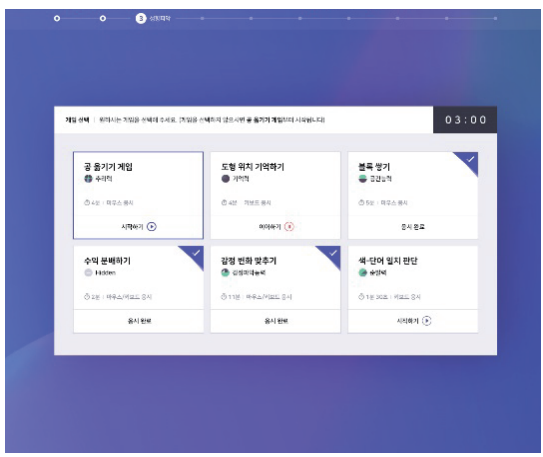

Brain science games

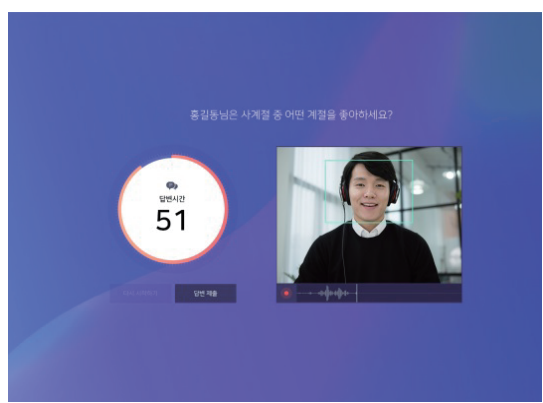

Interview questions

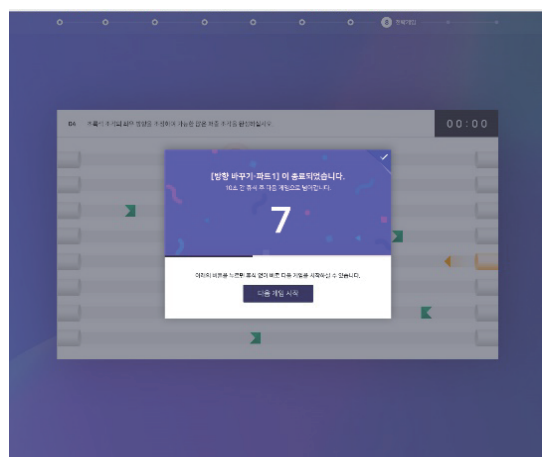

End of interview

Figure 3. Computer screen of the Al Interview process. Al, artificial intelligence.

Table 1. Detailed technology used in analysis of 4Vs: vision, voice, verbal, and vital sign

\begin{tabular}{|c|c|}
\hline Characteristic & Technology \\
\hline Vision & $\begin{array}{l}\text { - Technology that can recognize image information } \\
\text { - Imitation of human visual function } \\
\text { - Facial expressions, emotional expressions, eye movements, facial movements, etc. }\end{array}$ \\
\hline Voice & $\begin{array}{l}\text { - Technology that can recognize voice information } \\
\text { - Imitation of human auditory function } \\
\text { - Voice tone, size change, speed, pause change, pronunciation, etc. }\end{array}$ \\
\hline Verbal & $\begin{array}{l}\text { - Technology that can recognize language information } \\
\text { - Imitation of human language functions } \\
\text { - Speech to text (STT), Text to speech (TTS) } \\
\text { - Understand meaning of words, vocabulary usage, etc. }\end{array}$ \\
\hline Vital sign & $\begin{array}{l}\text { - Physiological data measurement technology } \\
\text { - Application of image information processing technology } \\
\text { - Pulse information detected by subtle changes in facial muscles and colors }\end{array}$ \\
\hline
\end{tabular}

discussed above. Instead of solving problems, the AI interview system has a total of 18 games that evaluate the motivation, emotional comprehension, cognitive abilities (e.g., executive function, attention control, memory, inference), decision styles, etc. of applicants (Figure 4). The game is configured differently depending on the position applied for, and each applicant solves different problems even if the applicants play the same type of games. The AI interview system generates questions within the same level of difficulty across different applicants. It is therefore difficult to disclose problems or cheat even if several applicants are interviewed at the same time.

Applicants respond to the game using the keyboard and mouse. Each game has clear goals, and feedback is provided as the game progresses. The system has access to cumulative data generated on the performance of tasks that would be difficult to grasp in face-to-face interviews or document evaluations. The game also allows the individual to reveal their unique response pattern because some questions may not have a particular correct answer. AI interviews can thus be more diverse and informative than regular aptitude tests with clear answers. While the 
[01] When you feel emotion in the person in the picture below, press the space bar and explain how you feel.

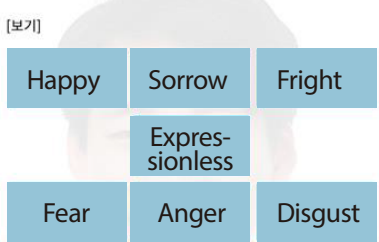

[001] Determine if the target meets the classifier.
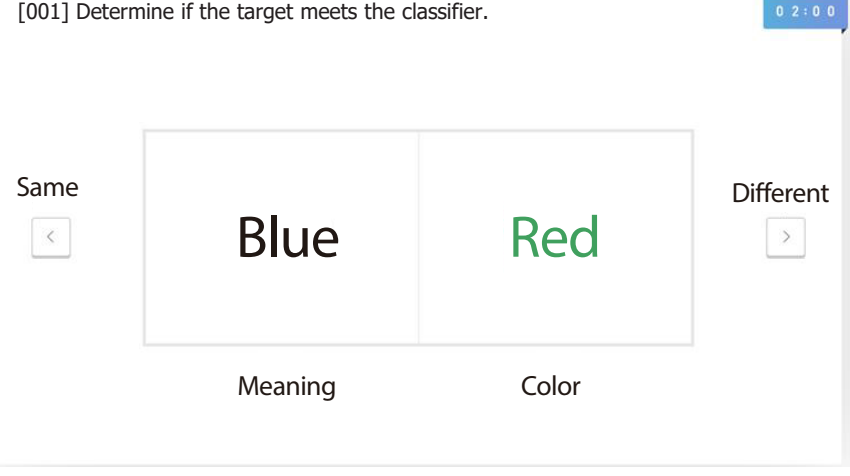

Determine if the figure on the screen matches the one from 4 steps earlier.

[01] Arrange the balls in heavy order.

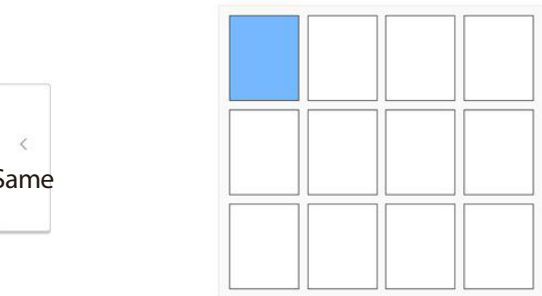

Different

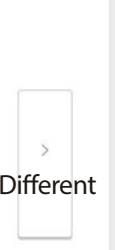

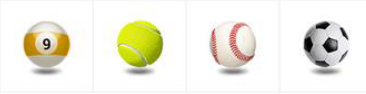

Submit

Figure 4. Computer screen shots of sample games.

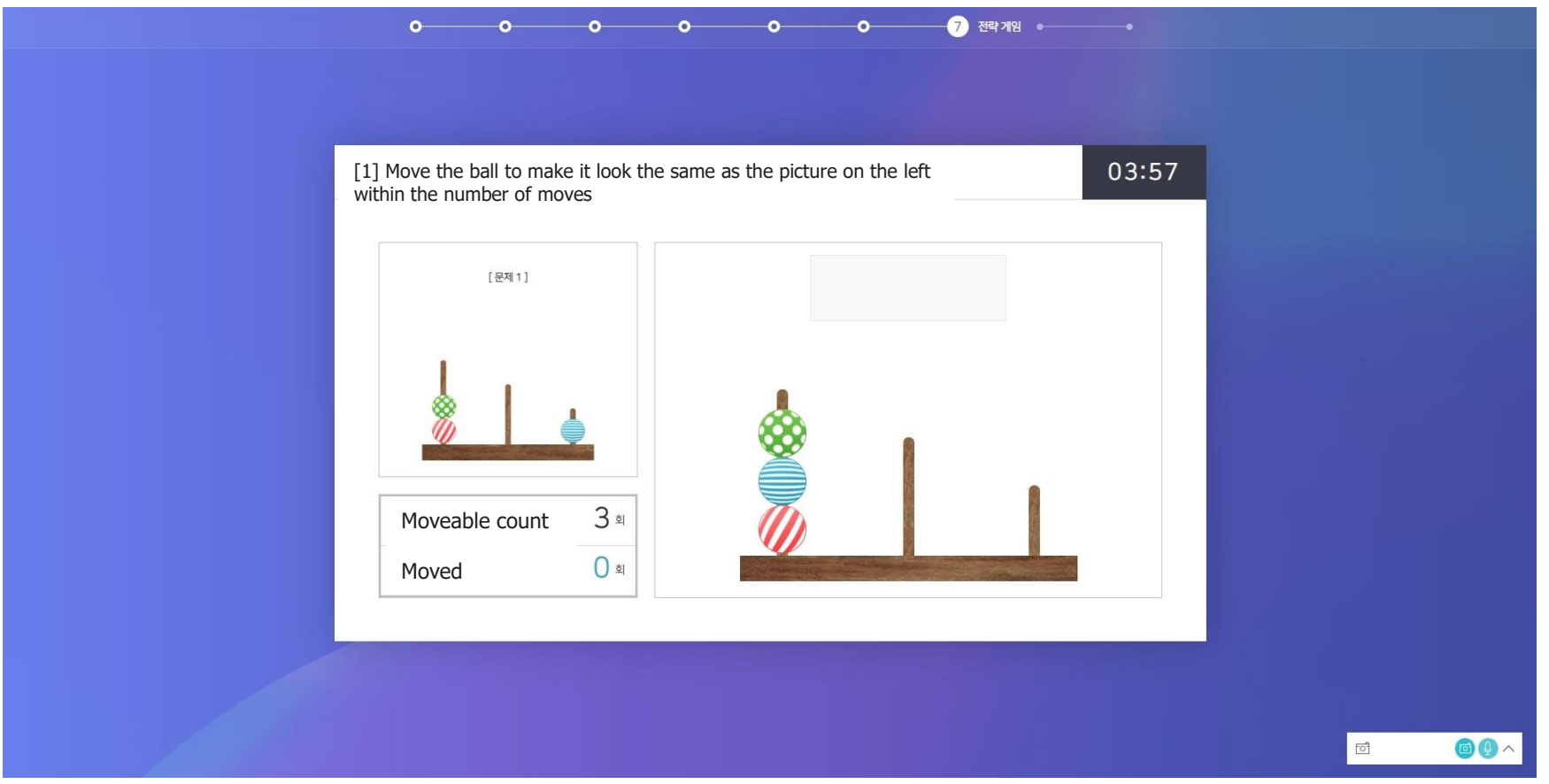

Figure 5. Sample game: Let's stack the ball.

applicant plays the game, the system collects and analyzes data such as response speed, response pattern, and learning curve.

For example, in a game called "Let's stack the ball," players must move balls onto three sticks within three turns to replicate a picture on the left (Figure 5). The player clicks the ball they want to move and then clicks the bar in which they wish 
to place the ball. The player cannot move balls more than three times. Questions become increasingly difficult when more questions are answered correctly. If an applicant makes a mistake, he or she can make up for it in the next session. Some players move the ball after a longer period of deliberation, while others decide their next moves as they are moving the ball. The AI interview system collects and analyzes all this information.

\section{Competency Evaluation and Prediction}

These AI interviews provide a detailed report of applicant characteristics extracted through games and surveys (Figure 6). This report provides information on the applicant's level of competence such as positivity, passion, cognitive function, emotional comprehension, and maladjustment in the workplace. It also predicts how outstanding the applicant will be through a model based on data of high performers in the field.

This information includes not only the suitability of the applicant for the particular position but also suggests other tasks that might be a good fit. If there is data on job interviews of current employees who have completed the AI interview, the report shows the incumbent who has competency profiles most similar to that of the applicant. The report also indicates how similar the applicant is to the high performers of the company.

The report then suggests questions that can be used in faceto-face interviews. These questions are generated based on the strengths and weaknesses of the applicant, which are analyzed by the AI interview. These questions are designed to help the interviewer assess the applicant more thoroughly in the face-toface interview process.
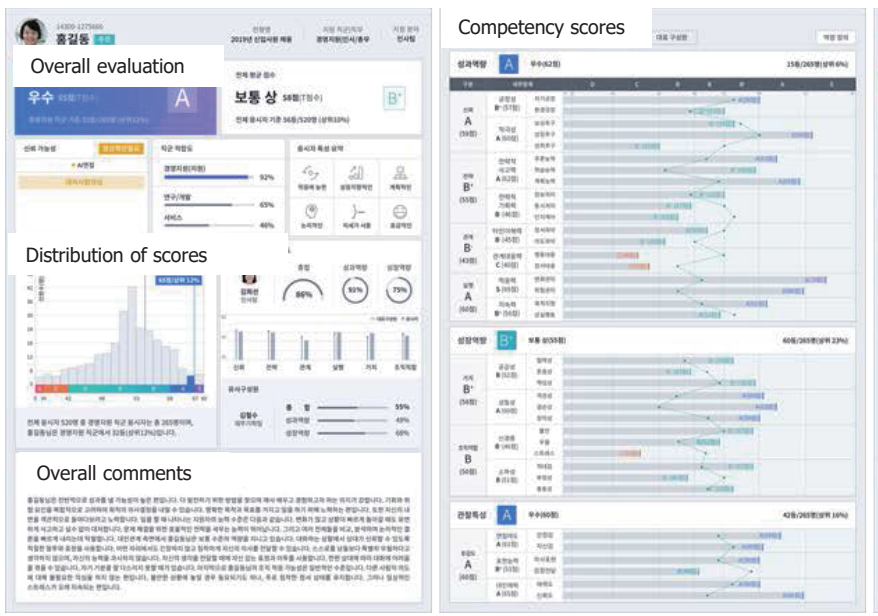

\section{Discussion}

\section{Effects of Al Interview}

The effectiveness of the use of $\mathrm{AI}$ in interviewing can be evaluated in terms of the applicant experience, the experience of $\mathrm{HR}$ managers responsible for recruitment, and the effectiveness of recruitment.

\section{Applicant Experience}

According to the survey of 3171 job seekers by Incruit, a job information portal (Jun, 2018), responses to AI recruitment showed that the applicants were $50.9 \%$ positive and $49.1 \%$ negative about the process. Positive responses came from the expectation that cheating can be verified, time and costs are saved, and corporate recruitment corruption can be addressed. Negative reactions were concerned AI's assessment of people itself and various personalities being evaluated with one standard. There were concerns regarding AI interviews: whether the candidate's ability and potential can be appropriately assessed, whether the machine would be able to properly evaluate various exceptions, and whether human intuition would have to be assessed. The prevailing opinion was that employers need to develop the AI recruitment process but do not know how.

The expectations and concerns of applicants for AI interviews are similar in content. The AI interview system is expected to evaluate more objectively, but it is also expected to imitate human bias. This concern may be due to the lack of sufficient information on $\mathrm{AI}$ interviews. According to an internal survey, games were considered fun and less burdensome. Those interviewed also said that they would be less likely to be influenced by problem-solving skills compared to the personality aptitude test.
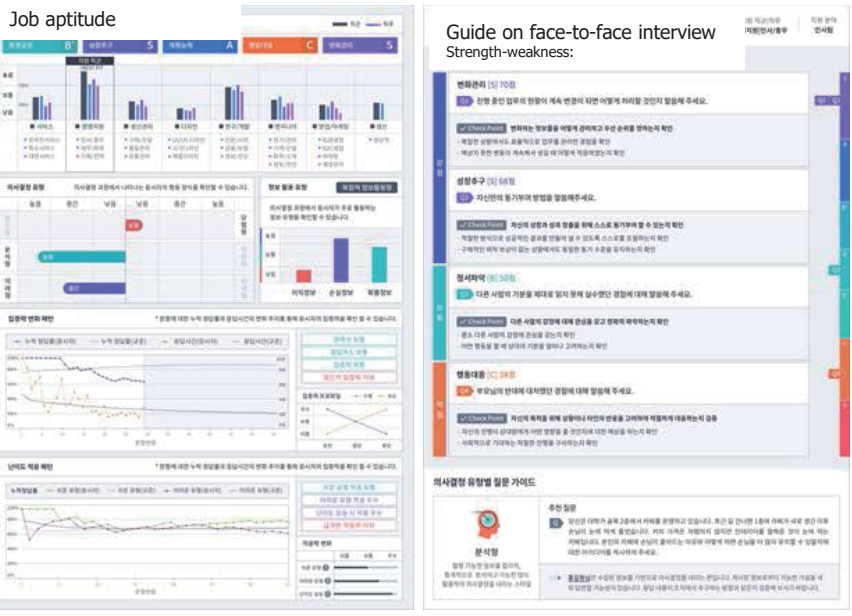

Figure 6. Sample report of results. 


\section{Experience of HR Managers in Charge of Recruitment}

HR managers' responses to AI interviews were generally positive. The points with which HR managers were most satisfied were that these interviews dramatically reduce the time and cost of recruitment and give them a chance to fully understand applicants before meeting the applicant. In particular, HR managers were highly satisfied when recruiting employees for customer-facing duties such as sales or customer service. This is because AI interviews can help them determine the levels of their performance on the key tasks required by the job. On the other hand, there were also concerns about the initial adoption, which may lead to an $\mathrm{AI}$ interview adversely affecting applicants of particular characteristics.

\section{Effectiveness of Recruitment}

The AI interview is a one-stop selection tool that includes document evaluations, personality assessments, and interviews. This is because it includes evaluation items in both documents and competency: self-introduction and application motivation for documents and games and surveys for competency. It is also an efficient tool in terms of the time required: An applicant takes about an hour to complete an $\mathrm{AI}$ interview, and the system takes less than an hour to analyze data from thousands of applicants.

\section{Future Direction of Al Interview}

MIDAS IT has a long-term vision for the AI interview system as a communication tool. First, the company aims to lessen the discomfort applicants feel with AI. To do so, the AI interview system should be able to interact with applicants in a more human way. For example, in a face-to-face interview process, if an interviewees see interviewers looking displeased or confused, they may realize that they are not doing very well in the interview and can try to change their approach to make up for their mistakes. With the AI interview process, however, the system does not give direct or indirect feedback to the applicants' responses, and applicants can thus feel anxious about not knowing anything about their performance. Anxiety can lead to negative experiences with the recruitment process and a distrust of the evaluation results. Thus, AI interviews should aim to provide more human interactions so applicants do not feel like they are speaking with a machine.

Second, adverse effects should be minimized in order to increase confidence in AI interviews. This does not mean that the AI interview system is currently having adverse effects. For example, AI interviews consistently collect data evenly so that there are no differences in treatment based on gender and continue to verify that no discrimination occurs. However, both HR managers and applicants are concerned about the adverse effects of interviews. The example of Amazon's AI hiring system algorithm, which was discarded by selectively evaluating women, is evidence that these concerns are legitimate (Dastin, 2018). As the objective of AI is to gather data on human decision-making, it is possible to learn human bias and conduct discrimination more efficiently. Therefore, in the process of AI interview development, we must continuously evaluate the various adverse effects of discrimination of race, gender, and age, and refine the data so that AI will learn more fairly. Companies should also focus on verifying how the tool has refined and learned the data and how the process was validated when choosing an $\mathrm{AI}$ interview tool.

Finally, the AI interview system aims to examine and provide insights into human psychology. After all, the effectiveness of AI interviews is directly related to what data is collected and learned. Unlike many other areas where AI is applied, the data used in recruitment is an assessment of people who fit the organization's culture or duties. As this assessment is made by a person, it contains a variety of psychological characteristics and variables. Developers of AI interviews and HR managers who want to use this tool must thus think more deeply about their organization. In this sense, AI interviews will not be a tool to replace HR manager, but rather a tool that will help HR managers consider more essential and core concerns.

\section{Conclusion}

In the HR field, AI has been adopted in the recruitment process in addition to processes evaluating employee experience, providing vocational training, and making decisions necessary for corporate management. The greatest value of the AI interview is that it significantly maximizes the communication channels between applicants and HR managers. The HR team can enjoy the benefit of essentially interviewing thousands of applicants in a short period of time. Applicants also have the opportunity to convey their passion, experience, and abilities to HR managers beyond what has been expressed in their documents. With the development of Internet and AI-based information processing technology, the communication channels between applicants and HR managers have been expanded.

\section{Conflict of Interest}

Hyejung Chang is an editor of Business Communication Research and Practice. However, she did not involve in the peer review evaluation and decision process of this article. Otherwise, no potential conflict of interest relevant to this article was reported. 


\section{References}

Bosco, F., Allen, D. G., \& Singh, K. (2015). Executive attention: An alternative perspective on general mental ability, performance, and subgroup differences. Personnel Psychology, 68(4), 859-898.

Cho, M. H. (2018, March 8). Midas IT launches AI recruitment solution. ZDNet. Retrieved from https://www.zdnet.com/article/ midas-it-launches-ai-recruitment-solution

Dastin, J. (2018, October 10). Amazon scraps secret AI recruiting tool that showed bias against women. Reuters. Retrieved from https://uk.reuters.com/article/us-amazon-com-jobs-automation-insight/amazon-scraps-secret-ai-recruiting-tool-thatshowed-bias-against-women-idUKKCN1MK08G

Denny, B. T., Kober, H., Wager, T. D., \& Ochsner, K. N. (2012). A meta-analysis of functional neuroimaging studies of self- and other judgments reveals a spatial gradient for mentalizing in medial prefrontal cortex. Journal of Cognitive Neuroscience, 24(8), 1742-1752.

Dorel, D., \& Aleksandra B. M. (2011). The role of information systems in human resource management. In A. Jean, D. Cvijanovic, \& J. Zubovic (Eds.), The role of labour markets and human capital in the unstable environment (pp. 25-46). Ploiesti, Romania: Karta Graphic.

Feloni, R. (2017, June 28). Consumer goods giant Unilever has been hiring employees using brain games and artificial intelligence - and it's a huge success. Business Insider. Retrieved from https://www.businessinsider.com/unilever-artificial-intelligencehiring-process-2017-6
Gershgorn, D. (2018, October 10). Amazon's "holy grail” recruiting tool was actually just biased against women. Quartz. Retrieved from https://qz.com/1419228/amazons-ai-powered-recruitingtool-was-biased-against-women

Higgins, D. M., Peterson, J. B., Pihl, R. O., \& Lee, A. G. M. (2007). Prefrontal cognitive ability, intelligence, big five personality, and the prediction of advanced academic and workplace performance. Journal of Personality and Social Psychology, 93(2), 298319.

Jun, J. H. (2018, April 17) More firms adopt AI-powered recruiting systems. The Korea Times. Retrieved from http://www.koreatimes. co.kr/www/tech/2018/04/129_247433.html

Lengnick-Hall, M. L., Lengnick-Hall, C. A., Andrade, L. S., \& Drake, B. (2009). Strategic human resource management: The evolution of the field. Human Resource Management Review, 19(2), 64-85.

Lewis, N. (2019, May 21). IBM transforms its approach to human resources with AI. SHRM. Retrieved from https://www. shrm.org/resourcesandtools/hr-topics/technology/pages/ ibm-transforms-human-resources-ai.aspx

Murayama, K., Matsumoto, M., Izuma, K., Sugiura, A., Ryan, R. M., Deci, E. L., \& Matsumoto, K. (2013). How self-determined choice facilitates performance: A key role of the ventromedial prefrontal cortex. Cerebral Cortex, 25(5), 1241-1251.

Sheopuri, A., \& Stachura, T. (2018, November 15). IBM's evolution of people analytics. WorkplaceTrends. Retrieved from https://workplacetrends.com/wp-content/uploads/2018/11/ Anshul-Sheopuri-and-Tom-Stachura-IBM.pdf 